\title{
Plasma membrane expression of ZNF185 is a prognostic factor in pancreatic ductal carcinoma
}

\author{
DAISUKE FURUKAWA ${ }^{1-3}$, TSUYOSHI CHIJIWA ${ }^{4}$, MASAHIRO MATSUYAMA $^{3}$, MASAYA MUKAI $^{5}$, \\ EI-ICHI MATSUO ${ }^{6}$, OSAMU NISHIMURA ${ }^{7}$, KENJI KAWAI $^{4}$, HIROSHI SUEMIZU $^{4}$, TOSHIO NAKAGOHRI ${ }^{3}$, \\ SOJI OZAWA ${ }^{3}$, KAZUAKI SHIMADA ${ }^{8}$, NOBUYOSHI HIRAOKA ${ }^{1,2}$ and MASATO NAKAMURA ${ }^{9}$ \\ Divisions of ${ }^{1}$ Pathology and Clinical Laboratories and ${ }^{2}$ Molecular Pathology, \\ National Cancer Center Research Institute, Tokyo $1040045 ;{ }^{3}$ Department of Surgery, \\ Tokai University School of Medicine, Isehara, Kanagawa 2591193; ${ }^{4}$ Central Institute for Experimental Animals, \\ Kawasaki, Kanagawa 2100821; ${ }^{5}$ Department of Surgery, Tokai University Hachioji Hospital, Hachioji, Tokyo 1920032; \\ ${ }^{6}$ Global Application Development Center, Shimadzu Corporation, Kyoto 6048511; Institute for Protein Research, \\ Osaka University, Suita, Osaka $5650871 ;{ }^{8}$ Division of Hepatobiliary and Pancreatic Surgery, \\ National Cancer Center Research Institute, Tokyo 1040045; ${ }^{9}$ Department of Regenerative Medicine, \\ Tokai University School of Medicine, Isehara, Kanagawa 2591193, Japan
}

Received June 29, 2016; Accepted March 9, 2017

DOI: $10.3892 / 01.2017 .6633$

\begin{abstract}
Pancreatic ductal carcinoma (PDC) is one of the major causes of cancer-associated mortality globally due to its high potential for distant metastasis. To understand hematogenous metastasis, the molecular expression profiles of weak metastatic PDC cell subline BxPC-3 and highly liver-metastatic cell subline LM-BxPC-3 were compared, and zinc finger protein 185 (ZNF185) was identified as a molecule that is upregulated in LM-BxPC-3 cells. The aim of the present study was to evaluate the clinicopathological significance of ZNF185 in PDC. Using immunohistochemistry, ZNF185 expression was investigated in 182 patients with PDC, in association with numerous clinicopathological variables. The expression profile of ZNF185 was also characterized using xenograft models. In contrast to parent BxPC-3 cells in subcutaneous transplanted tumor foci, which only expressed ZNF185 on their plasma membrane (m)ZNF185, LM-BxPC-3 cells in liver-metastatic foci that were formed subsequent to transplantation all expressed cytoplasmic (c)ZNF185. Additionally, 51\% of the cells at the periphery of the tumor foci expressed mZNF185. Expression of cZNF185, and of mZNF185 and cZNF185 combined was identified in 93 and 39\% of clinical patients with PDC, respectively. Patients with mZNF185-negative and -positive PDC exhibited a median survival time of 30.2 months and 21.3 months, respectively. Multivariate
\end{abstract}

Correspondence to: Dr Nobuyoshi Hiraoka, Division of Pathology and Clinical Laboratories, National Cancer Center Research Institute, 5-1-1 Tsukiji, Chuo, Tokyo 1040045, Japan

E-mail: nhiraoka@ncc.go.jp

Key words: zinc finger protein 185, pancreatic ductal carcinoma, prognosis, hematogenous metastasis, subcellular location analysis indicated that the expression of mZNF185 is closely associated with a shorter overall survival time. Increased marked venous invasion was more prevalent in patients who were mZNF185-positive, as compared with patients who were mZNF185-negative. These data suggest that the expression of mZNF185 is an independent and unfavorable prognosticator in patients with PDC. The results suggested that the amount and subcellular location of ZNF185 are correlated with the position of the cancer cells expressing it within the nests. Additionally, the subcellular location of ZNF185 may be important to its biological function.

\section{Introduction}

Pancreatic cancer (pancreatic ductal carcinoma; PDC) is one of the most devastating types of disease, as the early-stage tumors are difficult to detect but have a high potential for dissemination and distant metastasis $(1,2)$. A previous autopsy study revealed that $70 \%$ of patients succumb to the disease due to widespread metastasis, most frequently in the liver, peritoneum and lung, and that $30 \%$ succumb due to locally destructive disease (3). The control of hematogenous metastasis has been a long-standing concern for the prevention of cancer-associated mortality, but has yet to be achieved.

Chemotherapy regimens for PDC have improved over the past few years, but the prognosis for patients with metastatic pancreatic cancer remains poor, with an associated median survival time of 8-12 months (4-6). Surgical resection is the only definitive treatment available; however, the cancer recurs postoperatively in $80 \%$ of patients with PDC (7). For $\sim 20 \%$ of these patients with surgical resection, recurrence and mortality occurred within 6 months, and their survival time differed by several months compared with patients who could not undergo surgery due to pancreatic cancer metastasis (4-8). When lesions are identified preoperatively through diagnostic 
imaging, those indicative of local disease must be differentiated from occult distant metastases, which cause recurrence subsequent to surgery $(9,10)$; pancreatectomy may successfully treat local lesions, but offers little benefit to patients with metastatic disease. Local disease and metastatic disease are associated with different treatment regimens and survival times (4). If identified, a metastasis-specific biomarker may better indicate when resection is appropriate.

In order to understand the mechanisms underlying hematogenous metastasis in PDC, the molecular expression profile of the BxPC-3 PDC cell line, which demonstrates weak metastasis to the liver, was previously compared with that of the highly metastatic LM-BxPC-3 subline (11). This led to the isolation of ZNF185 as a metastasis-associated protein by global quantitative proteome analysis (12). ZNF185 contains two zinc-finger motifs in the $\mathrm{C}$-terminus that fit the consensus pattern of a LIM domain (13). The LIM-domain is a protein-protein interaction motif. A diverse group of proteins containing LIM domains have been identified with a range of functions. The majority of identified cytoplasmic LIM-domain proteins interact with the cytoskeleton or extracellular adhesion components to control cell morphology, motility and integrin-dependent adhesion and signaling (13). Since ZNF185 was observed to co-localize primarily with F-actin and partially with vinculin and paxillin, it was assumed that ZNF185 serves a role in cell adhesion (14).

At present, the biological and clinical significance of ZNF185 in cancer has not been investigated extensively. Only a small number of studies have focused on ZNF185 in the context of cancer: One group concluded that ZNF185 serves a tumor-suppressive role in prostate cancer (14), whereas another group considered ZNF185 to be an unfavorable prognostic indicator in colon cancer (12). In the present study, the clinicopathological significance of ZNF185 in PDC was investigated.

\section{Materials and methods}

Cells. The BxPC-3 human PDC cell line was purchased from the American Type Culture Collection (Manassas, VA, USA). A highly liver-metastatic cancer cell subline, LM-BxPC-3, was established via the intrasplenic transfer of cells derived from poorly developed but visible hepatic tumor foci formed following transplantation of the parental BxPC-3 cells and transfer to NOD/Shi-scid/IL-2R $\gamma^{\text {null }}(\mathrm{NOG})$ mice at the Central Institute for Experimental Animals (CIEA; Kanagawa, Japan) $(11,15,16)$. The cells were cultivated in RPMI-1640 medium (Sigma-Aldrich; Merck KGaA, Darmstadt, Germany) supplemented with $10 \%$ fetal bovine serum (BioWest, Nuaillé, France) in a humidified $\left(37^{\circ} \mathrm{C}, 5 \% \mathrm{CO}_{2}\right)$ incubator, and passaged on reaching $80 \%$ confluence.

Xenograft models. A total of 6 NOG mice (9-12 weeks of age, male) obtained from the CIEA were used in the present study. All animals were housed in plastic cages $(136 \times 208 \times 115 \mathrm{~mm})$ within a pathogen-free vinyl isolator system $(1,150 \times 500 \times 500 \mathrm{~mm})$ maintained at $22 \pm 1^{\circ} \mathrm{C}$ with $45 \pm 10 \%$ humidity, and there was a 12 -h light/dark cycle. To create subcutaneous models, $1 \times 10^{4} \mathrm{BxPC}-3$ tumor cells suspended in $0.1 \mathrm{ml}$ serum-free RPMI-1640 medium (Sigma-Aldrich; Merck KGaA) were injected into the subcutis of the mice. The experimental liver metastasis models were generated via intrasplenic injection of $1 \times 10^{4}$ LM-BxPC-3 cells in $0.1 \mathrm{ml}$ serum-free medium, followed by splenectomy after $1 \mathrm{~min}$, as previously described $(11,15,16)$. The mice were sacrificed under anesthesia and necropsied 6-8 weeks after inoculation, according to the standard protocols of the CIEA. Tumor tissues were surgically removed for standard histological examinations. The expression of ZNF185 in the tumors was evaluated using immunohistochemistry. Subsequent to immune-labeling as described in the immunohistochemical analysis section, the number of ZNF185-positive tumor cells per 1,000 tumor cells was counted in five distinct areas in each BxPC-3 subcutaneous tumor sample using an optical microscope at x200 magnification. Similarly, at the same magnification, the number of ZNF185-positive cells per 200 tumor cells were counted in 9 defined areas in the LM-BxPC-3 liver-metastatic tumor samples. The cells were counted separately in two areas of the cancer cell nests: Central and peripheral (Fig. 1). The peripheral area was defined as the outer two layers of cells in contact with the surrounding extracellular matrix, and the central area as the cells present in a central position within a cell nest, or the cells in areas other than the peripheral area. All experiments involving laboratory animals were performed in accordance with the care and use guidelines of the CIEA. The present study was approved by the Animal Committee of the CIEA (permit no. 08018A).

Study population. The present study included 182 patients who had undergone surgical resection for PDC at the National Cancer Center Hospital (NCC; Tokyo, Japan) between January 1990 and December 2005. All patients provided written informed consent. The patient cohort was comprised of 116 males and 67 females with a mean age of 64.1 years (range, 27-87 years). The pathological stages were classified according to the Union for International Cancer Control tumor-node-metastasis staging system (17). A total of 2 patients (1.0\%) were at Stage IA, $2(1.0 \%)$ at Stage IB, 30 $(16.5 \%)$ at Stage IIA, $129(70.9 \%)$ at Stage IIB and 19 (10.4\%) at Stage IV. No patient with Stage III disease involving the celiac artery or superior mesenteric artery underwent surgical resection. All patients with Stage IV disease were diagnosed on the basis of para-aortic lymph node metastasis. The clinicopathological features of the patients are summarized in Table I.

Pathological examination. Histological diagnosis was performed according to the WHO classification (2) and the Japan Pancreas Society Classification (18). Papillary adenocarcinoma, mucinous carcinoma and well-differentiated tubular adenocarcinoma were defined as well-differentiated (G1); moderately-differentiated tubular adenocarcinoma was defined as moderately-differentiated (G2); poorly-differentiated adenocarcinoma and adenosquamous carcinoma were defined as poorly-differentiated (G3). The following histopathological variables were evaluated according to the Japan Pancreas Society: Extra-pancreatic nerve plexus invasion, cancer-stroma association, lymphatic invasion, venous invasion and intrapancreatic nerve invasion. The grading of lymphatic invasion, venous invasion and 

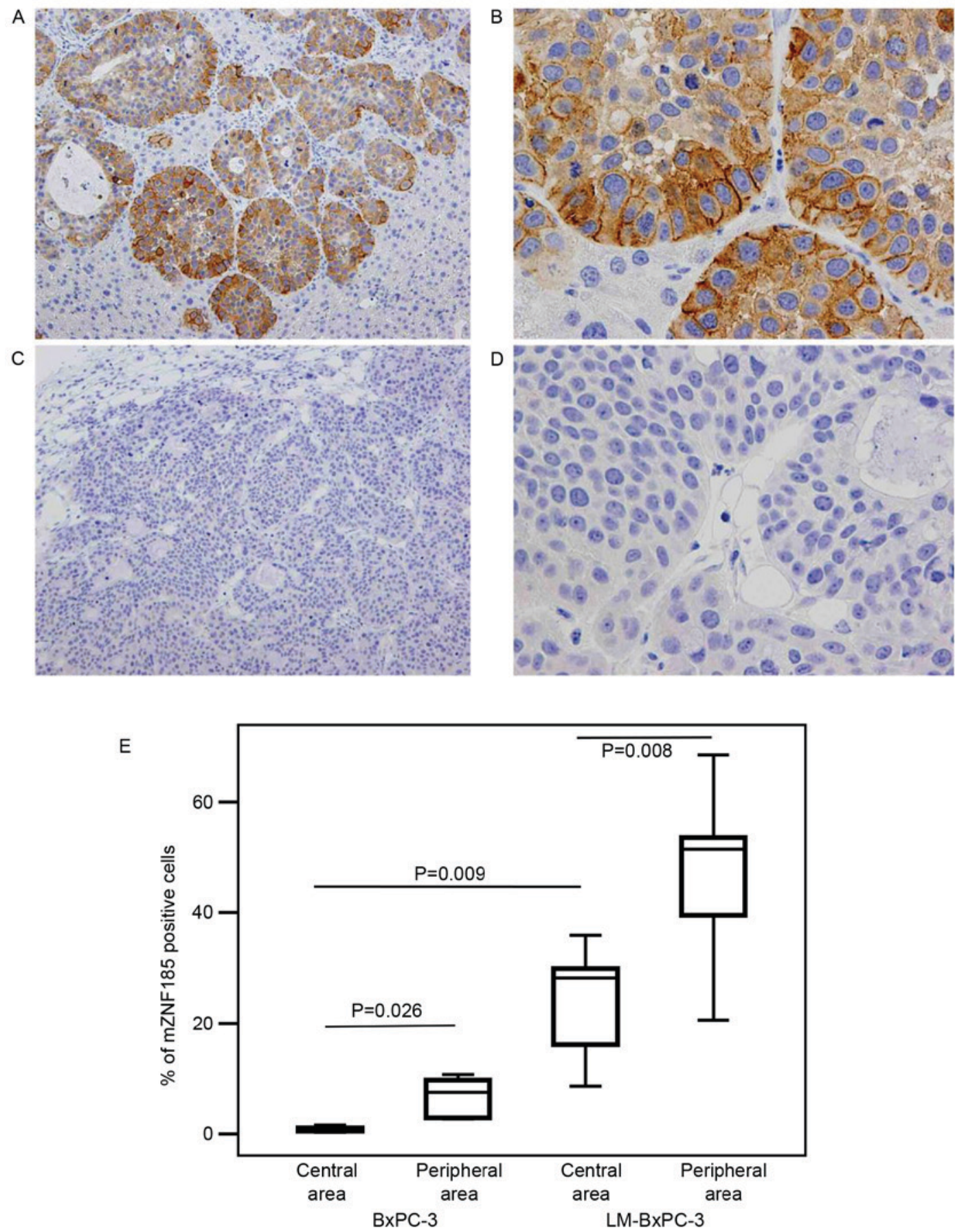

Figure 1. Immunohistochemical features of transplanted cancer cells. These representative images demonstrate: (A) x50 and (B) x200 magnification images of liver foci from the highly-metastatic LM-BxPC-3 cancer cell line; (C) x50 and (D) x200 magnification images of subcutaneous foci of the poorly-metastatic BxPC-3 cancer cell line. (E) A comparison of mZNF185-positive cancer cells. The percentage of mZNF185-immunopositive cells between subcutaneous tumor foci of the transplanted BxPC-3 cells and liver-metastatic tumor foci of the transplanted LM-BxPC-3 cells. These were also compared between the central and peripheral cells in the nests. The line in the middle of the boxes represents the median value. The bottom and top of the box indicate the 25 and 75 th percentile, respectively. The T-bars indicate the 95\% confidence interval. mZNF185, plasma membrane-associated zinc finger protein 185.

intrapancreaticnerveinvasion wasasdetermined asfollows:0,no invasion; 1 , slight invasion; 2 , moderate invasion; 3 , marked invasion.

Immunohistochemical analysis. Sections (4- $\mu$ m-thick) of representative formalin-fixed paraffin-embedded tissue blocks stained with a rabbit antibody specific to ZNF185 (Sigma-Aldrich; Merck KGaA) were used, as per the protocol of our previous study (12). The sections were incubated in
$0.3 \% \mathrm{H}_{2} \mathrm{O}_{2}$ in methanol at room temperature for $30 \mathrm{~min}$ to block endogenous peroxidase reaction. Subsequent to washing in PBS, sections were incubated with rabbit serum (Nichirei Biosciences Inc., Tokyo, Japan) at room temperature for $20 \mathrm{~min}$ to block non-specific binding. The sections were then incubated overnight in a humid chamber at $4^{\circ} \mathrm{C}$ with primary antibodies against ZNF-185 (dilution, 1:200; cat. no. HPA0004000; Sigma-Aldrich; Merck $\mathrm{KGaA}$ ). Following three washes in PBS, the sections were 
Table I. Correlation between the expression of ZNF185 on the plasma membrane and clinicopathological variables.

\begin{tabular}{|c|c|c|c|c|c|c|c|}
\hline \multirow{4}{*}{ Categories } & \multirow{2}{*}{\multicolumn{3}{|c|}{$\begin{array}{l}\text { ZNF185 expression on } \\
\text { the plasma membrane }\end{array}$}} & \multirow[b]{3}{*}{ Categories } & \multicolumn{3}{|c|}{$\begin{array}{l}\text { ZNF185 expression on } \\
\text { the plasma membrane }\end{array}$} \\
\hline & & & & & Negative & Positive & \\
\hline & \multirow{2}{*}{$\begin{array}{l}\text { Negative } \\
(n=111)\end{array}$} & \multirow{2}{*}{$\begin{array}{l}\text { Positive } \\
(\mathrm{n}=71)\end{array}$} & \multirow{2}{*}{ P-value } & & $(\mathrm{n}=111)$ & $(n=71)$ & P-value \\
\hline & & & & Stage (UICC) & & & \multirow{2}{*}{0.09} \\
\hline Age (years) & & & 0.84 & IA & 0 & 2 & \\
\hline$<65$ & 50 & 32 & \multirow{4}{*}{0.96} & IB & 1 & 1 & \\
\hline$\geq 65$ & 61 & 39 & & IIA & 23 & 7 & \\
\hline Gender & & & & IIB & 78 & 51 & \\
\hline Male & 70 & 45 & & IV & 9 & 10 & \\
\hline
\end{tabular}

\section{Location}

Pancreas head

Pancreas body/tail

Tumor size

$$
<30 \mathrm{~mm}
$$$$
\geq 30 \mathrm{~mm}
$$

Histologic grade

$\mathrm{G} 1$

G2/G3

Lymphatic invasion

$$
\text { ly0, } 1
$$$$
\text { ly } 2,3
$$

Venous invasion

v0, 1

v2, 3

Intra-pancreatic nerve invasion

$$
\text { ne } 0,1
$$

ne 2,3

Cancer-stroma

association

$$
\begin{aligned}
& \text { Medullary or } \\
& \text { Intermediate }
\end{aligned}
$$$$
\text { Scirrhous }
$$

Portal vein invasion

Negative

Positive

Extra-pancreatic nerve plexus invasion

$$
\text { Negative }
$$

Positive

Lymph node metastasis (UICC)

$$
\text { N0 }
$$

N1

Distant metastasis (UICC)
Table I. Continued.

${ }^{\mathrm{a}} \mathrm{P}<0.05, \chi^{2}$ test. $\mathrm{Ne}$, nerve invasion; UICC, Union for International Cancer Control; ZNF185, zinc finger protein 185. incubated with a peroxidase-labeled polymer-conjugated rabbit anti-goat antibody (Histofine Simple Stain Max PO; dilution, ready-to-use; cat. no. 414162F; Nichirei Biosciences, Inc.). The amplified immune products were visualized using the 3,3'-diaminobenzidine tetrahydrochloride reaction as described previously (19). The number of ZNF185-positive tumor cells was counted in 5 distinct areas using an optical microscope at x100 magnification. Quantification of ZNF185 expression was performed using a scale of negative, weakly positive, moderately positive and strongly positive; normal acinar cell staining was defined as moderately positive. Tissues that stained moderately-strongly positive in $>5 \%$ of the cells were defined as immunopositive.

Statistical analysis. The associations between ZNF185 expression levels and various clinicopathological parameters were evaluated statistically using the $\chi^{2}$ test. Survival curves were plotted according to the Kaplan-Meier method and statistical comparisons among the groups were performed using the log-rank test. Multivariate analysis of survival was conducted using the Cox proportional hazards model. All statistical analyses were performed using SPSS v. 21 (IBM SPSS, Armonk, $\mathrm{NY}$, USA). $\mathrm{P}<0.05$ was considered to indicate a statistically significant difference.

\section{Results}

$0.43 \quad$ Unique distribution of ZNF185 expression in pancreatic cancer cells. LM-BxPC-3 cells were established as a highly liver-metastatic subline derived from the poorly-metastatic BxPC-3 cells, and appeared to express ZNF185 more abundantly compared with the parental cells. When the expression of ZNF185 was examined in xenotransplanted tumors in immunocompromised NOG mice, it was identified that the subcellular location of ZNF185 varied depending on the position of the cancer cells expressing it in the cancer cell nests (Fig. 1). ZNF185 was expressed in the cytoplasm of $76.1 \pm 15.5 \%$ of the parental cells located at the periphery of the cancer cell nests, whereas only $5.8 \pm 2.4 \%$ of the parent cancer cells at the center of the nests expressed cytoplasmic ZNF185. A 

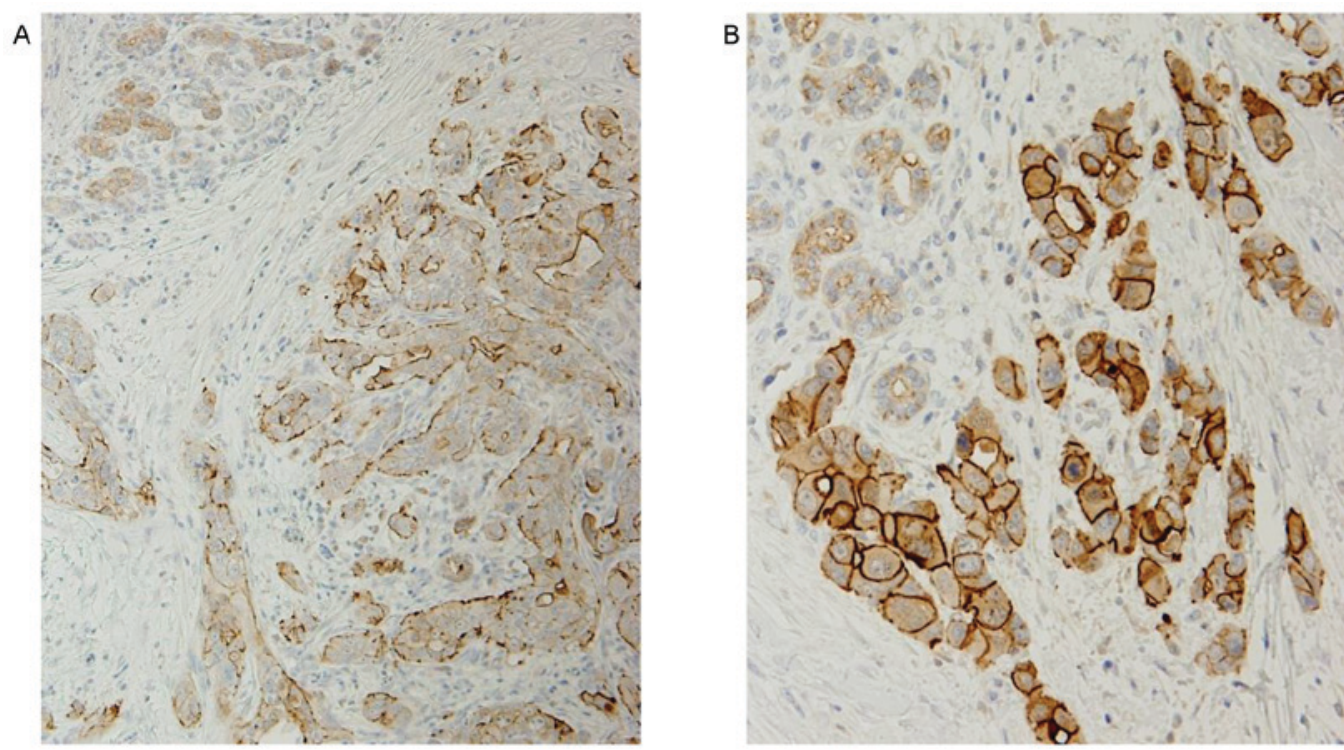

Figure 2. Immunohistochemical features of pancreatic ductal carcinoma. (A) Adenocarcinoma cells are immunopositive for ZNF185 in membranous and cytoplasmic patterns, particularly in the plasma membrane of cancer cells attached to the stroma. Non-cancerous ductular cells also express ZNF185 (top left). Magnification, x50. (B) Adenocarcinoma cells strongly express ZNF185 in the plasma membrane and cytoplasm. Magnification, x100. ZNF185, zinc finger protein 185 .

A

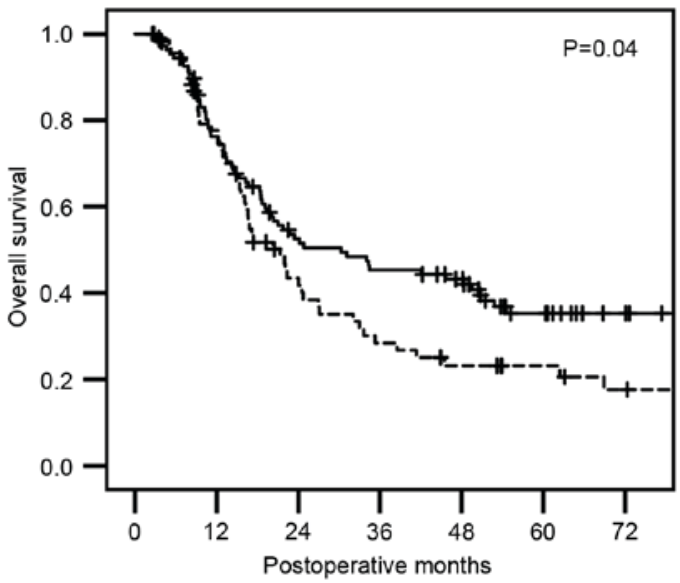

B

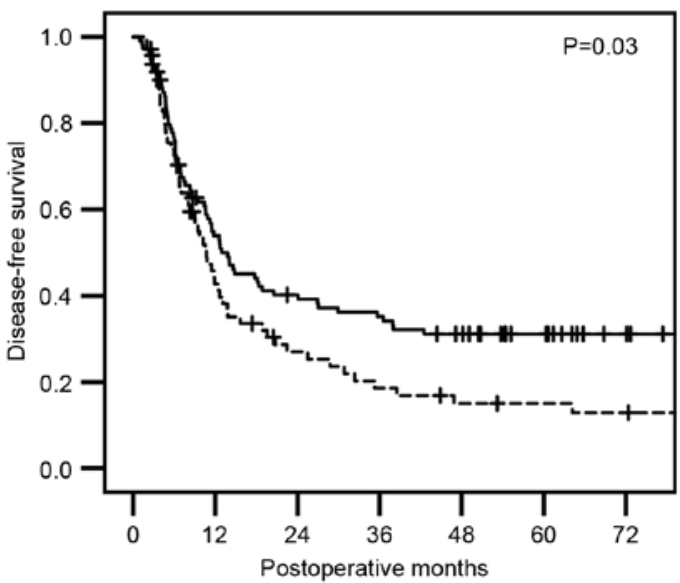

Figure 3. Kaplan-Meier survival curves comparing the (A) overall survival and (B) disease-free survival between the groups, indicating the positive (dotted line) and negative (solid line) expression of mZNF185. ${ }^{*} \mathrm{P}<0.05$ from the log-rank test. mZNF185, plasma membrane-associated zinc finger protein 185. small population of the cells expressed ZNF185 on the plasma membrane, representing $6.7 \pm 3.7$ and $0.8 \pm 0.5 \%$ of the parent cancer cells located at the periphery and center of the nests, respectively (Fig. 1). By contrast, all of the LM-BxPC-3 cells expressed cytoplasmic ZNF185 irrespective of their position in the cancer cell nests; $47.0 \pm 14.2$ and $24.4 \pm 9.6 \%$ of the peripheral and central cells, respectively, demonstrated membrane expression of ZNF185 (Fig. 1). These data suggest that the amount and subcellular location of ZNF185 are correlated with the position of the cells expressing it within the cancer cell nests, and also that expression of ZNF185 on the plasma membrane is associated with hematogenous metastasis. The clinicopathological significance of the characteristic pattern of ZNF185 expression in PDC was subsequently investigated.

Expression of ZNF185 in pancreatic cancer tissues. In clinical samples of PDC tissues, ZNF185 was identified to be expressed in the cytoplasm and on the plasma membrane of cancer cells. ZNF185 expression was observed in the cytoplasm of cancer cells (cZNF185) in 169/182 patients with PDC (92.8\%), and in the cytoplasm and plasma membrane (mZNF185) in 71 patients $(39 \%)$. None of the patients with PDC exhibited expression of ZNF185 exclusively on the plasma membrane of the cancer cells (Fig. 2). ZNF185 was also expressed in non-neoplastic acinar cells of the pancreatic exocrine glands.

Significantly shorter survival time for PDC patients with mZNF185-positive cancer cells. Patients with PDC with and without mZNF185-positive cancer cells exhibited a median overall survival (OS) time of 21.3 months [95\% confidence interval (CI)], 15.9-26.7 months) and 30.2 months (95\% CI, 12.1-48.4 months), respectively, and a disease-free survival (DFS) time of 10.7 months (95\% CI, 7.9-13.4 months) and 12.9 months (95\% CI, 7.9-13.4 months), respectively. Kaplan-Meier survival analyses revealed that patients with mZNF185-positive cancer demonstrated a significantly shorter OS (P=0.04; Fig. $3 \mathrm{~A})$ and 
Table II. Univariate and multivariate analyses of various clinicopathological factor and ZNF185 expression for overall survival and disease-free survival.

A, Overall survival

\begin{tabular}{|c|c|c|c|c|}
\hline & \multicolumn{2}{|c|}{ Univariate analysis } & \multicolumn{2}{|c|}{ Multivariate analysis } \\
\hline & $\begin{array}{l}\text { Hazard ratio } \\
(95 \% \mathrm{CI})\end{array}$ & P-value & $\begin{array}{l}\text { Hazard ratio } \\
\quad(95 \% \mathrm{CI})\end{array}$ & P-value \\
\hline Age $(\geqq 65)$ & $0.82(0.57-1.19)$ & 0.31 & & \\
\hline Gender (male) & $1.23(0.83-1.81)$ & 0.29 & & \\
\hline Location (pancreas head) & $1.18(0.80-1.74)$ & 0.39 & & \\
\hline Size $(\geqq 30 \mathrm{~mm})$ & $2.11(1.40-3.17)$ & $<0.01^{\mathrm{a}}$ & & \\
\hline Histologic type (G2, G3) & $1.57(1.01-2.42)$ & $0.04^{\mathrm{a}}$ & & \\
\hline Stroma (scirrhous) & $0.88(0.60-1.30)$ & 0.54 & & \\
\hline Lymphatic invasion (ly2, ly3) & $2.65(1.66-4.13)$ & $<0.01^{\mathrm{a}}$ & $2.46(1.51-3.82)$ & $<0.01^{\mathrm{a}}$ \\
\hline Venous invasion $(\mathrm{v} 2, \mathrm{v} 3)$ & $2.00(1.35-2.96)$ & $<0.01^{\mathrm{a}}$ & & \\
\hline Intrapancreatic neural invasion (ne2, ne 3 ) & $1.66(1.14-2.43)$ & $0.008^{\mathrm{a}}$ & & \\
\hline Portal vein invasion (present) & $1.98(1.34-2.86)$ & $<0.01^{\mathrm{a}}$ & $1.73(1.20-2.51)$ & $<0.01^{\mathrm{a}}$ \\
\hline Nerve plexus invasion (present) & $1.47(1.01-2.13)$ & $0.04^{\mathrm{a}}$ & & \\
\hline Lymph node metastasis (N1) & $1.91(1.12-3.25)$ & $0.01^{\mathrm{a}}$ & & \\
\hline Distant metastasis (M1) & $1.30(0.71-2.37)$ & 0.39 & & \\
\hline $\begin{array}{l}\text { ZNF185 expression } \\
\text { (positive on the plasma membrane) }\end{array}$ & $1.45(1.008-2.11)$ & $0.04^{\mathrm{a}}$ & $2.19(1.04-2.19)$ & $0.02^{\mathrm{a}}$ \\
\hline
\end{tabular}

B, Disease-free survival

\begin{tabular}{|c|c|c|c|c|}
\hline Age $(\geq 65)$ & $0.90(0.64-1.28)$ & 0.580 & & \\
\hline Gender (male) & $1.14(0.79-1.64)$ & 0.460 & & \\
\hline Location (pancreas head) & $1.07(0.74-1.55)$ & 0.694 & & \\
\hline Size $(\geq 30 \mathrm{~mm})$ & $2.16(1.47-3.17)$ & $<0.001^{\mathrm{a}}$ & & \\
\hline Histologic type (G2, G3) & $1.48(0.99-2.20)$ & 0.054 & & \\
\hline Stroma (scirrhous) & $0.68(0.47-0.99)$ & $0.049^{\mathrm{a}}$ & $0.68(0.46-1.004)$ & 0.053 \\
\hline Lymphatic invasion (ly2, ly3) & $1.98(1.32-2.96)$ & $0.001^{\mathrm{a}}$ & $1.70(1.12-2.57)$ & $0.012^{\mathrm{a}}$ \\
\hline Venous invasion (v2, v3) & $2.15(1.48-3.12)$ & $<0.001^{\mathrm{a}}$ & $1.66(1.13-2.45)$ & $0.009^{\mathrm{a}}$ \\
\hline Intrapancreatic neural invasion (ne2, ne 3 ) & $1.62(1.13-2.31)$ & $0.008^{\mathrm{a}}$ & & \\
\hline Portal vein invasion (present) & $1.45(1.02-2.05)$ & $0.035^{\mathrm{a}}$ & & \\
\hline Nerve plexus invasion (present) & $1.68(1.18-2.39)$ & $0.004^{\mathrm{a}}$ & & \\
\hline Lymph node metastasis (N1) & $2.29(1.35-3.88)$ & $0.002^{\mathrm{a}}$ & $1.90(1.12-3.24)$ & $0.017^{\mathrm{a}}$ \\
\hline Distant metastasis (M1) & $1.81(1.08-3.03)$ & $0.023^{\mathrm{a}}$ & & \\
\hline $\begin{array}{l}\text { ZNF185 expression } \\
\text { (positive on the plasma membrane) }\end{array}$ & $1.44(1.01-2.04)$ & $0.039^{\mathrm{a}}$ & $1.43(1.003-2.05)$ & $0.048^{\mathrm{a}}$ \\
\hline
\end{tabular}

${ }^{\mathrm{a}} \mathrm{P}<0.05$. ZNF185, zinc finger protein $185 ; \mathrm{CI}$, confidence interval.

a significantly shorter DFS ( $\mathrm{P}=0.03$; Fig. $3 \mathrm{~B})$, as compared with patients with mZNF185-negative cancer. There was no significant difference in OS or DFS observed between patients with and without cZNF185-positive cancer.

Prognostic significance of mZNF185 expression in association with clinicopathological factors. Univariate analysis indicated that tumor size, histologic grade, lymphatic invasion, venous invasion, intra-pancreatic nerve invasion, portal vein invasion, extra-pancreatic nerve plexus invasion, nodal metastasis and the expression of mZNF185 were all factors significantly correlated with a shorter OS time. Subsequent multivariate analysis of these factors indicated that lymphatic invasion, portal vein invasion and the expression of mZNF185 were significant (Table IIA). With regard to DFS, univariate analysis indicated that tumor size, tumor-stroma ratio, lymphatic invasion, venous invasion, intrapancreatic nerve invasion, portal vein invasion, intra-pancreatic nerve invasion, nodal metastasis, distant metastasis and the expression of mZNF185 were factors significantly correlated with a shorter DFS time. Multivariate 
analysis indicated that tumor-stroma ratio, lymphatic invasion, venous invasion, lymph node metastasis and the expression of mZNF185 were all significant (Table IIB). Among the various clinicopathological variables, only marked venous invasion was more prevalent in patients who were mZNF185-positive, as compared with in patients who were mZNF185-negative (Table I).

\section{Discussion}

PDC has a typically poor prognosis due to its aggressive behavior and extensive invasion and metastasis, particularly hematogenous metastasis $(1,2)$. ZNF185 has been isolated as a metastasis-associated protein by global quantitative proteome analysis of the BxPC-3 and LM-BxPC-3 PDC cell lines $(11,15)$. In the present study, the expression of ZNF185 in PDC was characterized clinicopathologically.

Firstly, using xenograft models, the unique distribution of the ZNF185 molecule in cancer cells was observed. In contrast to the tumor foci formed by the poorly metastatic parent cells, of which only a small number expressed ZNF185 on their cell membrane, the liver metastatic foci that formed subsequent to the transplantation of highly metastatic cells expressed cytoplasmic ZNF185 in all patients, and half of the cells present at the periphery of cancer cell nests also expressed ZNF185 on their cell membranes. It is suggested that the amount and subcellular location of ZNF185 are correlated with the position of the cancer cells expressing it within cell nests. As the expression of mZNF185 was limited to cells at the periphery of the cancer cell nests that were in direct contact with the surrounding environment, it is assumed that mZNF185 may serve a role in tumor behavior, including motility.

Next, the distribution of ZNF185 in patients with PDC was clinicopathologically investigated and it was identified that mZNF185, but not cZNF185, was an unfavorable prognostic factor in OS and DFS. Only marked venous invasion was significantly correlated with mZNF185 expression among the various clinicopathological variables examined (Table I). When the rates of hematogenous metastasis (to the liver, lung, bone, pleura and adrenal glands) following surgical resection were analyzed in the same PDC cohort, it was identified to occur more frequently in patients with mZNF185-positive cancer $[51.7 \%(31 / 60)]$ as compared with in patients without mZNF185-positive cancer [37.9\% (39/103) odds ratio; 1.82 ; 95\% CI, 0.92-3.60; $\mathrm{P}=0.081]$. It has been suggested that higher expression levels of ZNF185 in colon cancer are significantly associated with an increased frequency of liver metastasis and are an indicator of poor prognosis (14). These data suggest that higher expression levels of mZNF185 are associated with accelerated hematogenous spreading of PDC. The occurrence of distant metastasis was not determined to be correlated with mZNF185 expression, as all patients with distant metastasis in the present study only exhibited distant lymph node metastasis, and not other types of distant metastasis (Table I).

It has been suggested that, in prostate cancer cells, ZNF185 is co-localized primarily with F-actin and partially with paxillin and vinculin (14). Paxillin and vinculin are components of the cell matrix adhesion apparatus $(20,21)$. Focal adhesion, which provides a structural link between cells and the extracellular matrix, also facilitates signal transduction for the mediation of various biological processes (22). Focal adhesion involves numerous kinases, adaptors and cytoskeletal proteins. The dynamics of focal adhesion turnover is a key regulatory determinant of cancer cell migration, invasion and metastasis (23). Paxillin binds to numerous proteins involved in the organization of the actin cytoskeleton, which are necessary for motility events associated with tumor metastasis (20). The overexpression of paxillin has been suggested to be associated with distant metastasis and poor prognosis in colorectal cancer (24), salivary adenoid cystic carcinoma (25) and hepatocellular carcinoma (26). Vinculin has also been implicated in cell invasion and metastasis (21). It is speculated that ZNF185 serves a role in the acceleration of cancer invasion and metastasis, in association with focal adhesion. In our previous study, it was suggested that patients who expressed ZNF185 also exhibited a poor prognosis in cases of colon cancer (12). To the best of our knowledge, only one previous study has indicated that ZNF185 functions as a tumor-suppressive protein through an association with actin cytoskeletal dynamics in prostate cancer (14). The present study identified that mZNF185 was localized not only between cells and the extracellular matrix, but also between cells that were present only in the peripheral areas of cell nests (Fig. 1). Further prospective studies must additionally investigate the involvement of mZNF185 in hematogenous metastasis in PDC.

In conclusion, the expression of mZNF185 is hypothesized to be an independent indicator of unfavorable prognosis in patients with PDC, being particularly evident in highly liver-metastatic cancer cells located at the periphery of cancer cell nests in xenograft models. It is also suggested that mZNF185 may be involved in cancer cell motility, particularly hematogenous metastasis, and that the amount and the subcellular location of ZNF185 are each correlated with the location of the cells expressing it in the cell nests.

\section{Acknowledgements}

This study was supported by a grant-in-aid for Scientific Research to Daisuke Furukawa (type C; grant no. 16K07096) and Masato Nakamura (type B; grant no. 15H04287) from the Ministry of Education, Culture, Sports, Science and Technology, Japan.

\section{References}

1. Hidalgo M: Pancreatic cancer. N Engl J Med 362: 1605-1617, 2010.

2. Hruban RH, Boffetta P, Hiraoka N, Iacobuzio-Donahue C, Kato Y, Kern SE, Klimstra DS, Kloppel G, Maitra A, Offerhaus GJA, et al: Ductal Adenocarcinoma of the Pancreas. In: World Health Organization Classification of Tumours. Pathology \& Genetics. Tumours of the Digestive System. Bosman FT, Carneiro F, Hruban RH and Theise ND (eds) IARC, Lyon, pp281-291, 2010.

3. Iacobuzio-Donahue $\mathrm{CA}, \mathrm{Fu} \mathrm{B}$, Yachida S, Luo M, Abe $\mathrm{H}$, Henderson CM, Vilardell F, Wang Z, Keller JW, Banerjee P, et al: DPC4 gene status of the primary carcinoma correlates with patterns of failure in patients with pancreatic cancer. J Clin Oncol 27: 1806-1813, 2009.

4. Ueno H, Ioka T, Ikeda M, Ohkawa S, Yanagimoto H, Boku N, Fukutomi A, Sugimori K, Baba H, Yamao K et al: Randomized phase III study of gemcitabine plus S-1, S-1 alone, or gemcitabine alone in patients with locally advanced and metastatic pancreatic cancer in Japan and Taiwan: GEST study. J Clin Oncol 31: $1640-1648,2013$ 
5. Von Hoff DD, Ervin T, Arena FP, Chiorean EG, Infante J, Moor M, Seay T, Tjulandin SA, Ma WW, Saleh MN, et al: Increased survival in pancreatic cancer with nab-paclitaxel plus gemcitabine. N Engl J Med 369: 1691-1703, 2013.

6. Conroy T, Desseigne F, Ychou M, Bouché O, Guimbaud R, Bécouarn Y, Adenis A, Raoul JL, Gourgon-Bourgade S, de la Fouchardiére C, et al: FOLFILINOX versus gemcitabine for metastatic pancreatic cancer. N Engl J Med 364: 1817-1825, 2011.

7. Oettle H, Neuhaus P, Hochhaus A, Hartmann JT, Gellert K, Ridwelski K, Niedergethmann M, Zülke C, Fahlke J, Arning MB, et al: Adjuvant chemotherapy with gemcitabine and long-term outcomes among patients with resected pancreatic cancer: The CONKO-001 randomized trial. JAMA 310 : 1473-1481, 2013.

8. Groot VP, Rezaee N, Wu W, Cameron JL, Fisgman EK, Hruban RH, Weiss MJ, Zheng L, Wolfgang CL and He J: Patterns, timing, and predictors of recurrence following pancreatectomy for pancreatic ductal adenocarcinoma. Ann Surg: Mar 23, 2017 (Epub ahead of print)

9. Yokoyama N, Otani T, Hashidate H, Maeda C, Katada T, Sudo N, Manabe S, Ikeno Y, Tuyoda A and Katayanagi N: Real-time detection of hepatic micrometastases from pancreatic cancer by intraoperative fluorescence imaging: Preliminary results of a prospective study. Cancer 118: 2813-2819, 2012.

10. Satoi S, Yanagimoto H, Yamamoto T, Toyokawa H, Hirooka S, Yamaki S, Opendro SS, Inoue K, Michiura T, Ryota H, et al: A clinical role of staging laparoscopy in patients with radiographically defined locally advanced pancreatic ductal adenocarcinoma. World J Surg Oncol 14: 14, 2016.

11. Suemizu H, Monnai M, Ohnishi Y, Ito M, Tamaoki N and Nakamura M: Identification of a key molecular regulator of liver metastasis in human pancreatic carcinoma using a novel quantitative model of metastasis in NOD/SCID $/ \gamma_{c}$ null (NOG) mice. Int J Oncol 31: 741-751, 2007.

12. Furukawa D, Chijiwa T, Matsuyama M, Mukai M, Matsuo EI, Nishimura O, Kawai K, Suemizu H, Hiraoka N, Nakagohri T, et al: Zinc finger protein 185 is a liver metastasis-associated factor in colon cancer patients. Mol Clin Oncol 2: 709-713, 2014

13. Zheng Q and Zhao Y: The diverse biofunctions of LIM domain proteins: Determined by subcellular localization and protein-protein interaction. Biol Cell 99: 489-502, 2007.

14. Zhang JS, Gong A and Young CY: ZNF185, an actin-cytoskeleton-associated growth inhibitory LIM protein in prostate cancer. Oncogene 26: 111-122, 2007.
15. Hamada K, Monnai M, Kawai K, Nishime C, Kito C, Miyazaki N, Ohnishi Y, Nakamura M and Suemizu H: Liver metastasis models of colon cancer for evaluation of drug efficacy using NOD/Shi-scid IL2R $\gamma$ null (NOG) mice. Int J Oncol 32: 153-159, 2008.

16. Matsuyama M, Wakui M, Monnai M, Mizushima T, Nishime C, Kawai K, Ohmura M, Suemizu H, Hishiki T, Suematsu M, et al: Reduced CD73 expression and its association with altered purine nucleotide metabolism in colorectal cancer cells robustly causing liver metastases. Oncol Lett 1: 431-436, 2010.

17. Sobin LH, Gospodarowicz MK and Wittekind C: TNM Classification of Malignant Tumours. Wiley-Blackwell, Hoboken, NJ, 2011.

18. Japan-Pancreas-Society: Classification of Pancreatic Cancer. Kanehara, Tokyo, Japan, 2011.

19. Oguro S, Ino Y, Shimada K, Hatanaka Y, Matsuno Y, Esaki M, Nara S, Kishi Y, Kosuge T and Hiraoka N: Clinical significance of tumor-infiltrating immune cells focusing on BTLA and Cbl-b in patients with gallbladder cancer. Cancer Sci 106: 1750-1760, 2015.

20. Schaller MD: Paxillin: A focal adhesion-associated adaptor protein. Oncogene 20: 6459-6472, 2001.

21. Mierke CT: The role of vinculin in the regulation of the mechanical properties of cells. Cell Biochem Biophys 53: 115-126, 2009.

22. Yam JW, Tse EY and Ng IO: Role and significance of focal adhesion proteins in hepatocellular carcinoma. J Gastroenterol Hepatol 24: 520-530, 2009.

23. Nagano M, Hoshino D, Koshikawa N, Akizawa T and Seiki M: Turnover of focal adhesions and cancer cell migration. Int J Cell Biol 2012: 310616, 2012.

24. Zhao CJ, Du SK, Dang XB and Gong M: Expression of paxillin is correlated with clinical prognosis in colorectal cancer patients. Med Sci Monit 21: 1989-1995, 2015.

25. Shi J, Wang S, Zhao E, Shi L, Xu X and Fang M: Paxillin expression levels are correlated with clinical stage and metastasis in salivary adenoid cystic carcinoma. J Oral Pathol Med 39: 548-551, 2010.

26. Li HG, Xie DR, Shen XM, Li HH, Zeng H and Zeng YJ: Clinicopathological significance of expression of paxillin, syndecan-1 and EMMPRIN in hepatocellular carcinoma. World $\mathbf{J}$ gastroenterol 11: 1445-1451, 2005 